\title{
Effects of Time Delays in a Dynamical Heart Model
}

\author{
Ricardo Páez-Hernández ${ }^{1 *}$, Maribel Zamora-Gómez ${ }^{2}$, Pedro Portillo-Díaz ${ }^{1}$, Delfino Ladino-Luna ${ }^{1}$ and Rubén \\ Luévano-Enríquez ${ }^{1}$ \\ 1. Basic Sciences Department, Metropolitan Autonomous University Campus Azcapotzalco, Federal District 02200, México \\ 2. Hospital General “Dr Gaudencio González Garza” La Raza, IMSS, Federal District 02290, México
}

Received: December 28, 2013 / Accepted: January 17, 2014 / Published: March 25, 2014.

\begin{abstract}
In this work, a dynamical model of the heartbeat is studied using nonlinear dynamics and considering the time delays inherent in the system. Two fixed points are associated to sustained oscillations which might be interpreted as the diastole and systole. These parameters are associated with blood flow in human body called arterial pressure and are very important in the cardio-vascular diagnostic.
\end{abstract}

Key words: Heartbeat, systole, diastole, stability, steady state.

\section{Introduction}

The heart is a complicate but robust pump. It has four valves. There are two circuits for the blood, one which spreads through the lungs to pick up oxygen and the other which spreads through the body to deliver the oxygenated blood. The first circuit is a low-pressure one so as not to damage the delicate membrane in the lungs, and the second is a high-pressure circuit so that the blood gets all the way down to the feet and up again.

During the heart beat cycle there are two extreme equilibrium states, namely diastole which is the relaxed state and systole which is the contracted state. What makes the heart beat is the presence of a pacemaker which is located on the top of atrium.

The pacemaker causes the heart to contract into systole. That is, it triggers off an electrochemical wave which spreads slowly over the atria causing the muscle fiber to contract and push blood into ventricles, and then spreads rapidly over the ventricles, causing the whole ventricle to contract into systole and deliver a big pump of blood down the arteries. The muscle fibers

\footnotetext{
*Corresponding author: Ricardo Páez Hernández, Ph.D., research fields: finite time thermodynamics and non-linear dynamics. E-mail: phrt@correo.azc.uam.mx.
}

then rapidly relax and return the heart to a diastole; then the process is repeated.

\section{Non-linear Dynamics}

As is it known, the heart muscle is an autonomous system and has an intermittent dynamic, i.e., its operation is periodic. Then, the authors use non-linear dynamics considering the time delays inherent to the heart to study the model heart's dynamics proposed by Zeeman [1].

\subsection{Time Delays}

In real life situations, when the value of a variable is modified the effect in the dynamic response of the system is not observed immediately. A certain time must elapse until the system begins to respond or "feel" the effect of the changes made. Suppose we modify the concentration of a reactor feed. The authors' experience, and common sense tells us that time passes until the variables that characterize the dynamic behavior of the reactor (e.g., concentration) begin to modify its value relative to their pre-change. These systems are known as dynamical systems. Delayed systems appear naturally in medicine, biology and engineering. These systems have been studied since 
before the last century. Studies in Medicine and Biology begin with Ross' epidemiology models (1911) and others in the early twentieth century, which were studied by McDonald [2]. A distinctive feature of these systems is that their rate of evolution is described by differential equations that include information about the history of the system. The effects of delays are of great interest, since their presence may include complex behavior (oscillations, instability, bad system performance). Páez-Hernández and Santillán [3] studied the effect that time delays produce in a mathematical model for the stretch reflex regulatory pathway. Rojas-Pacheco et al. [4] studied time-delay effects on the dynamics of a two-actor conflict model.

\subsection{Fixed Points and Linearization System with Delays}

Consider a dynamic system which has a single variable with time delays $\xi$,

$$
\begin{aligned}
& \frac{d x}{d t}=f\left(x, y_{\xi}\right) \\
& \frac{d y}{d t}=g\left(x_{\xi}, y\right)
\end{aligned}
$$

Subscript $\xi$ is a time delay variable. Following step by step the Strogatz procedure, in order to obtain a linear system [5].

$$
\dot{u}=f\left(x^{*}, y^{*}+v_{\xi}\right), \dot{v}=g\left(x^{*}+u_{\xi}, y^{*}+v\right)
$$

where, $u$ and $v$ represent a small perturbation of the system and $\left(x^{*}, y^{*}\right)$ is a fixed point, now the authors do a Taylor's series expansion to Eq. (3) and the authors consider negligible the terms of two on ward, and evaluate in the steady-state and the authors obtain:

$$
\begin{aligned}
& \dot{u}=\left.\frac{\partial f}{\partial x}\right|_{\left(x^{*}, y^{*}\right)} u+\left.\frac{\partial f}{\partial y_{T}}\right|_{\left(x^{*}, y^{*}\right)} v_{\xi} \\
& \dot{v}=\left.\frac{\partial g}{\partial x_{T}}\right|_{\left(x^{*}, y^{*}\right)} u_{\xi}+\left.\frac{\partial g}{\partial y}\right|_{\left(x^{*}, y^{*}\right)} v
\end{aligned}
$$

Now, the authors assume that $u$ and $v$ are of the form:

$$
\begin{gathered}
u=A_{1} e^{\lambda t} \\
u_{\xi}=A_{1} e^{\lambda t} e^{-\lambda \xi} \\
v=A_{2} e^{\lambda t} \\
v_{\xi}=A_{2} e^{\lambda t} e^{-\lambda \xi}
\end{gathered}
$$

where, $\lambda$ is a complex number, $A_{1}$ and $A_{2}$ are constant.
Substituting Eqs. (6)-(9) into Eqs. (4) and (5) leads to the following set of homogeneous linear system for $A_{1}$ and $A_{2}$ :

$$
\begin{aligned}
& \left(f_{x}-\lambda\right) A_{1}+f_{y_{\xi}} e^{-\lambda \xi} A_{2}=0 \\
& f_{x_{\xi}} e^{-\lambda \xi} A_{1}+\left(g_{y}-\lambda\right) A_{2}=0
\end{aligned}
$$

This system of equations has non-trivial solutions only if the determinant of the matrix of coefficients equals zero, i.e.,

$$
\left(f_{x}-\lambda\right)\left(g_{y}-\lambda\right)-f_{x_{\xi}} g_{y_{\xi}} e^{-2 \lambda \xi}=0
$$

This equation is also called the transcendental characteristic equation, and can be written as:

$$
H(z)+K(z) e^{-z \xi}=0
$$

being $z$ an eigenvalue, and $H(z)$ and $K(z)$ are second and zero order polynomials, respectively.

The solutions to this equation are not obvious because has an infinite number of roots [2]. One way to overcome this situation is to consider the fact a common effect of time delays to destabilize stable fixed points or to stabilize unstable fixed points by sustained oscillations. If we assume that $(z=i \omega)$, and substitute in Eq. (13), the authors obtain a complex variable equation.

$$
P(\omega)+i Q(\omega)=e^{-i \omega \xi}
$$

where, $P(\omega)$ and $Q(\omega)$ are second and first order polynomials, respectively. The authors observe that the right hand side of this equation represents the unitary circle whereas the left hand side describes a parabola. The intersection of these two curves could represent a change in the stability of the system. The analysis of intersection between the parabola and the unitary circle leads to the following classification:

If the parabola does not intersect the unitary circle, and the system is stable at $\xi=0$, then the system is stable independent of delay.

If the system is stable for $\xi=0$ and the parabola intersects the unit circle, then the system can be affected by delays.

\subsection{Non-linear Dynamics Model of Heart}

A mathematical model that describes the behavior of the heartbeat was developed in Ref. [1], where it was suggested that such a model contain three basic 
features:

(1) A stable equilibrium state representing diastole;

(2) The threshold for triggering the electrochemical wave causing the heart to go into systole;

(3) The return of the heart into the diastolic state.

The resulting model is given by

$$
\begin{gathered}
\dot{x}=-\frac{1}{\epsilon}\left(x^{3}-T x+y\right) \\
\dot{y}=x+x_{d}
\end{gathered}
$$

where $x(t)$ represents the length of the muscle fiber, $y(t)$ is a variable related to electrochemical activity; the parameter $\epsilon$ is a small positive constant associated to the local stability of an endoreversible system with the fast eigenvalue of the system, $x_{d}$ is a scalar quantity representing a typical length of muscle fiber in the diastolic state, and $T$ represents tension in the muscle fiber. Now we use the result for linearization systems, for the case $\xi=0$,

$$
f(x, y)=-\frac{1}{\epsilon}\left(x^{3}-T x+y\right)
$$

and

$$
g(x, y)=x+x_{d}
$$

so this expression yields:

$$
\begin{aligned}
\left.f_{x}\right|_{\left(x^{*}, y^{*}\right)} & =\frac{T}{\epsilon} \\
\left.f_{y}\right|_{\left(x^{*}, y^{*}\right)} & =-\frac{1}{\epsilon} \\
\left.g_{x}\right|_{\left(x^{*}, y^{*}\right)} & =1 \\
\left.g_{y}\right|_{\left(x^{*}, y^{*}\right)} & =0
\end{aligned}
$$

Now, substituting Eqs. (19)-(22) into Eq. (12), the authors obtain the eigenvalues $\lambda_{1}=3.62$ and $\lambda_{2}=1.38$ for $T=1, \epsilon=0.2$ and $x_{d}=0$. Therefore, the origin is unstable since both eigenvalues are real and positive. In Fig. 1, the authors show the phase portrait of Eqs. (1) and (2), with the same values for the parameters, the cubic line (red curve) represents the steady state of Eq. (18), $A$ and $B$ may be represent the systole and diastole points.

\section{Dynamics Effects of Time Delays}

Consider again the systems of delay differential equations, given by Eqs. (2) and (3), but now $\xi=\frac{\pi}{2}$. They can rewritten as:

$$
\begin{aligned}
& \frac{d x}{d t}=f\left(x, y_{\pi / 2}\right) \\
& \frac{d y}{d t}=g\left(x_{\pi / 2}, y\right)
\end{aligned}
$$

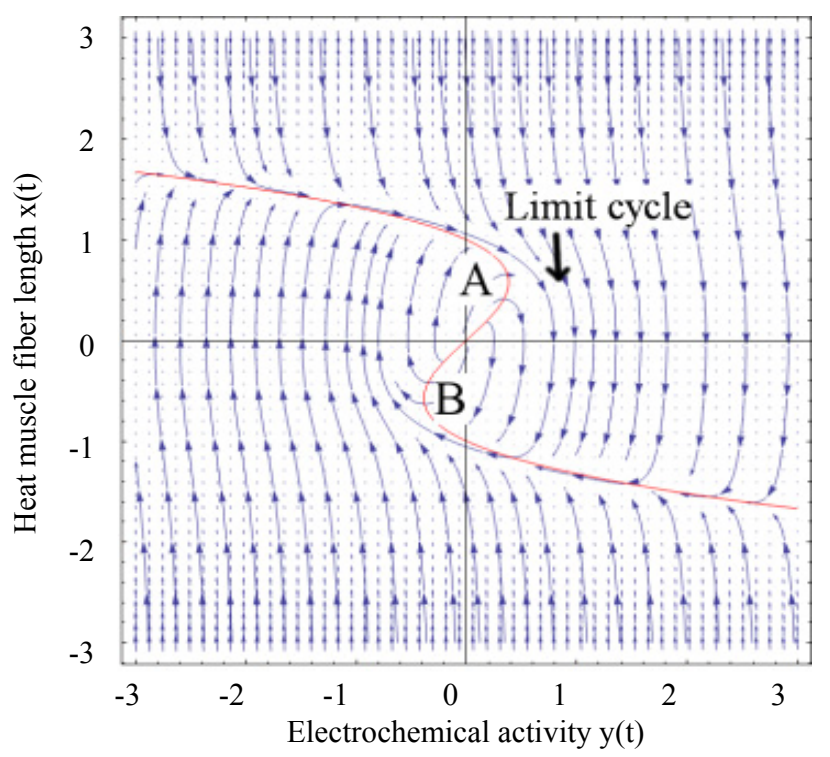

Fig. 1 Phase portrait of the heartbeat model.

with $f$ and $g$ as defined in Eqs. (17) and (18). Following Section 2.1, the time course of small perturbations from the steady state is determined; we can write Eq. (12) as:

$$
\left(f_{x}-\lambda\right)\left(g_{y}-\lambda\right)-f_{y} g_{x} e^{-\lambda \pi}=0
$$

The stability analysis of a dynamic system involving time delays can be quite complicated due to the fact that, in general, the characteristic equation has an infinite number of solutions. On the other hand, it is known that a common effect of time delays is to destabilize formerly stable steady states by inducing sustained oscillations. To test whether this happens, assume that $\lambda$ is imaginary $(\lambda=i \omega)$ and substitute into the characteristic equation to obtain

$$
\left(-E \omega^{2}+F\right)+i D \omega=e^{(-i \omega \pi)}
$$

with

$$
E=\frac{1}{f_{y} g_{x}}, F=\frac{f_{x} g_{y}}{f_{y} g_{x}} \text { and } D=\frac{f_{x}+g_{y}}{f_{y} g_{x}}
$$

It follows from Eqs. (19)-(22) that $f_{x}>0, f_{y}<0$, $g_{x}>0$ and $g_{y}=0$. This further implies that $E<0, F$ $=0$ and $D>0$.

The left-hand side of Eq. (26) determines the lower branch of a horizontal parabola in the complex plane. This parabola opens to the right and its vertex is located in the $(0,0)$ point. On the other hand, the right-hand side of Eq. (26) determines a unitary circle in the 
complex plane. The points where these curves cross correspond to values of $\omega$ and $\pi$ at which sustained oscillations appear due to a destabilization of the steady state, or vice versa. Let $\rho$ and $\sigma$ real variables along the real and the imaginary axes of the complex plane, respectively. In terms of these variables, the equation for the parabola can be written as:

$$
\rho=-\frac{E}{D^{2}} \sigma^{2}
$$

While the equation for the circle is:

$$
\rho^{2}+\sigma^{2}=1
$$

To find the points where both curves cross, solve for $\sigma$ in Eq. (28) and substitute into Eq. (28) to obtain:

$$
\rho^{2}-\frac{D^{2}}{E} \rho=0
$$

The solutions for the last equation give the real coordinates of the crossing points. The corresponding imaginary coordinates can be then calculated as $\sigma=-\sqrt{1-\rho^{2}}$. The solutions of Eq. (29) are:

$$
\begin{aligned}
& \rho_{1}=\frac{L}{2}+\frac{1}{2} \sqrt{L^{2}-4 K} \\
& \rho_{2}=\frac{L}{2}-\frac{1}{2} \sqrt{L^{2}-4 K}
\end{aligned}
$$

with $L=D^{2} / E$ and $K=-1$. From its definition and because $E$ is negative and $D$ is positive, $L$ is negative. Notice that $\rho_{1}$ and $\rho_{2}$ have common points.

Therefore, the parabola of Eq. (27) crosses the unitary circle in the points $(-0.19 \pm 0.98)$, this indicating that there a two points which induce oscillations, i.e., those points can be destabilize the system. Fig. 2, shows the intersections between unit circle and the parabola given in Eqs. (27) and (28).

\section{Conclusions}

From the point of view of the authors the non-linear dynamics theory is an useful and powerful tool to deal with systems displaying time delays. Because it was possible to get two points which destabilize the system, which are represented by the intersections showed in Fig. 2, the authors think that such points might be

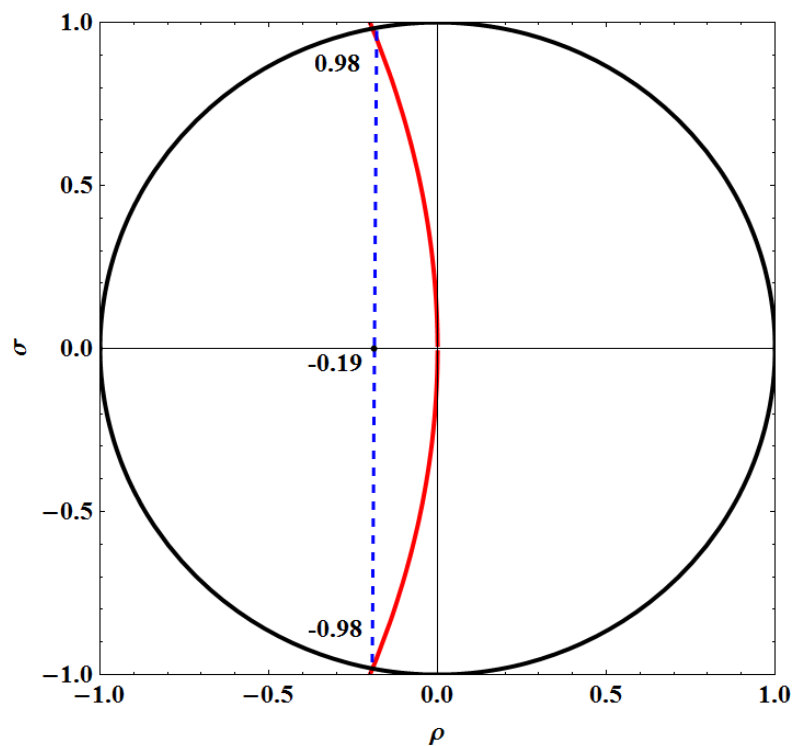

Fig. 2 Plot shows the intersections between unit circle and the parabola given in Eqs. (27) and (28).

associated to the arterial pressure states, both the systole and diastole.

\section{Acknowledgments}

This work was partially supported by CONACYT (SNI).

\section{References}

[1] Zeeman, E. C. Differential Equations for Heartbeat and Nerve Impulse. Toward a Theoretical Biology 1972, 4, 8-67.

[2] McDonald, N. Biological Delay Systems: Linear Stability Theory; Cambridge University Press: Cambridge, 1989.

[3] Páez-Hernández, R.; Santillán, M. Comparison of the Energetic Properties and the Dynamical Stability in a Mathematical Model of the Stretch Reflex. Physica A 2008, 387, 3574-3582.

[4] Rojas-Pacheco, A.; Obregón-Quintana, B.; Liebovitch, L. S.; Guzmán-Vargas, L. Time-Delay Effects on Dynamics of a Two-Actor Conflict Model. Physica A 2013, 392, 458-467.

[5] Strogatz, H. S. Non Linear Dynamics and Chaos: With Applications to Physics, Chemistry and Engineering; Perseus: Cambridge, MA, 1994. 